\title{
Parametric amplification of a superconducting plasma wave
}

\author{
S. Rajasekaran ${ }^{1,2}$, E. Casandruc ${ }^{1,2}$, Y. Laplace ${ }^{1,2}$, D. Nicoletti ${ }^{1,2}$, G. D. Gu ${ }^{3}$, S. R. Clark ${ }^{4,5}$, D. Jaksch ${ }^{5,6}$ \\ and A. Cavalleri ${ }^{1,2,5 \star}$
}

Many applications in photonics require all-optical manipulation of plasma waves', which can concentrate electromagnetic energy on sub-wavelength length scales. This is difficult in metallic plasmas because of their small optical nonlinearities. Some layered superconductors support Josephson plasma waves $^{2,3}$, involving oscillatory tunnelling of the superfluid between capacitively coupled planes. Josephson plasma waves are also highly nonlinear ${ }^{4}$, and exhibit striking phenomena such as cooperative emission of coherent terahertz radiation ${ }^{5,6}$, superconductor-metal oscillations ${ }^{7}$ and soliton formation ${ }^{8}$. Here, we show that terahertz Josephson plasma waves can be parametrically amplified through the cubic tunnelling nonlinearity in a cuprate superconductor. Parametric amplification is sensitive to the relative phase between pump and seed waves, and may be optimized to achieve squeezing of the order-parameter phase fluctuations ${ }^{9}$ or terahertz single-photon devices.

Cuprates are strongly anisotropic superconductors in which transport is made three-dimensional by Josephson tunnelling between the $\mathrm{Cu}-\mathrm{O}$ planes. Tunnelling reduces the superfluid density in the direction perpendicular to the planes, and hence the frequency of the plasmon to below the average pair breaking gap. Weakly damped oscillations of the superfluid sustain transverse Josephson plasma waves (JPWs) that propagate along the planes.

Consider a complex superconducting order parameter in the $i$ th $\mathrm{Cu}-\mathrm{O}$ plane $\psi_{i}(x, y, t)=\left|\psi_{i}(x, y, t)\right| \exp i \theta_{i}(x, y, t)$, which depends on two in-plane spatial coordinates $x$ and $y$ and on time $t$. For a terahertz-frequency optical field polarized perpendicular to the planes, excitations above the superconducting gap are negligible and the modulus of the order parameter $|\psi|^{2}$ (number of Cooper pairs) is nearly constant in space and time. Hence, the electrodynamics is dominated by the order-parameter phase $\theta_{i}(x, y, t)$. Ignoring at first the spatial dependence of the phase, the local tunnelling strength can, from the Josephson equation $\mathrm{s}^{10}$, be expressed in terms of an equivalent inductance, $L$, which depends on the local interlayer phase difference $\theta_{i, i+1}(t)=\theta_{i}(t)-\theta_{i+1}(t)$ as $L\left(\theta_{i, i+1}(t)\right) \sim L_{0} / \cos \left(\theta_{i, i+1}(t)\right)$ ( $i$ and $i+1$ are the indices for two neighbouring layers). Here $L_{0}=\hbar / 2 e I_{c}$ is the inductance at equilibrium, $\hbar$ the reduced Planck's constant, $2 e$ the Cooper pair charge and $I_{\mathrm{c}}$ the critical current. Denoting the capacitance of the $\mathrm{Cu}-\mathrm{O}$ planes with a constant $C$, we express the Josephson plasma resonance (JPR) frequency as $\omega_{\mathrm{IP}}^{2}=1 /\left(L\left(\theta_{i, i+1}(t)\right) C\right)=\omega_{\mathrm{IP} 0}^{2} \cos \left[\theta_{i, i+1}(t)\right]$, where $\omega_{\mathrm{IP} 0}^{2}=1 / L_{0} C$ is the equilibrium value. Correspondingly, the oscillator strength $f \sim \omega_{\mathrm{IP}}^{2}$ for the plasma oscillations ${ }^{11}$ is also a function of the interlayer phase, and scales as $f=f_{0} \cos \left[\theta_{i, i+1}(t)\right]$.

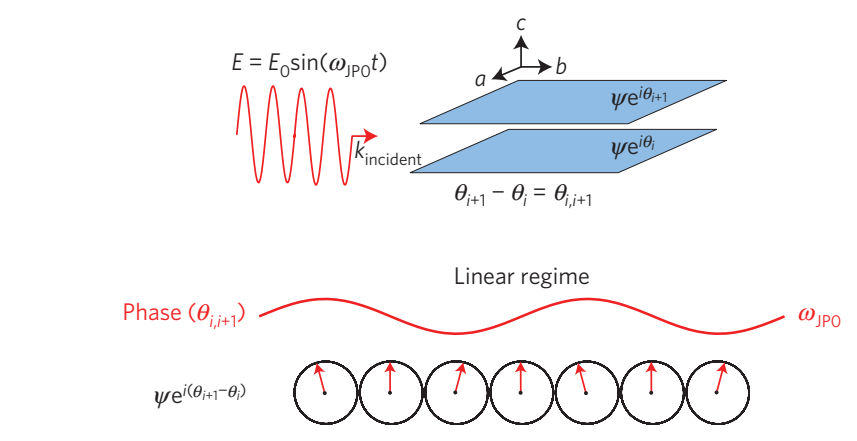

Oscillator strength $(f) \longrightarrow$

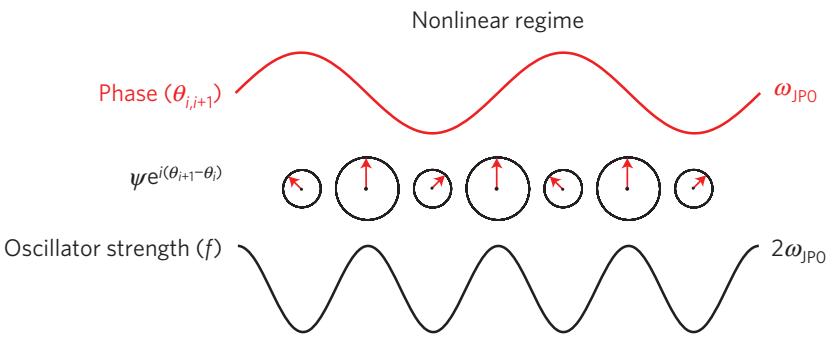

Figure 1 | Schematic representation of Josephson plasma waves. Schematic time-dependent representation of JPWs in the linear and nonlinear regime in the presence of a driving field $E(t)=E_{0} \sin \left(\omega_{\mathrm{Jpo}} t\right)$ polarized along the out-of-plane direction of a layered superconductor. Red arrows indicate the Josephson phase, while the corresponding oscillator strength $f$ is represented by the black circle area. A JPW in the linear regime consists of small-amplitude modulations of $\theta_{i, i+1}$ at constant oscillator strength $f \sim \omega_{\mathrm{JPO}}^{2}$. In the nonlinear regime, the Josephson phase oscillates at $\omega \mathrm{JPO}$, whereas $f$ is modulated at $2 \omega_{\mathrm{JPO}}$.

The dependence of the oscillator strength $f$ on the cosine of the superconducting phase corresponds to a third-order optical nonlinearity.

According to the second Josephson equation ${ }^{10}$, the interlayer phase difference $\theta_{i, i+1}(t)$ advances in time with the time integral of the interlayer voltage drop, as $\partial\left[\theta_{i, i+1}(t)\right] / \partial t=2 \mathrm{eV} / \hbar$. For an optical field made resonant with the Josephson plasma frequency $E(t)=E_{0} \sin \left(\omega_{\mathrm{JP} 0} t\right)$, the interlayer phase oscillates as $\theta_{i, i+1}(t)=\theta_{0} \cos \left(\omega_{\mathrm{JP} 0} t\right)$, where $E_{0}$ is the field amplitude and $\theta_{0}=\left(2 e d / \hbar \omega_{\mathrm{IPO}}\right) E_{0} \quad(d \sim 1 \mathrm{~nm}$ is the interlayer distance $)$. This

\footnotetext{
${ }^{1}$ Max Planck Institute for the Structure and Dynamics of Matter, Luruper Chaussee 149, 22761 Hamburg, Germany. ${ }^{2}$ Center for Free-Electron Laser Science, Luruper Chaussee 149, 22761 Hamburg, Germany. ${ }^{3}$ Condensed Matter Physics and Materials Science Department, Brookhaven National Laboratory, Upton, New York 11973-5000, USA. ${ }^{4}$ Department of Physics, University of Bath, Claverton Down, Bath BA2 7AY, UK. ${ }^{5}$ Department of Physics, Oxford University, Clarendon Laboratory, Parks Road, Oxford OX1 3PU, UK. ${ }^{6}$ Centre for Quantum Technologies, National University of Singapore, 3 Science Drive 2, Singapore 117543, Singapore. ${ }^{\star} e-m a i l:$ andrea.cavalleri@mpsd.mpg.de
} 
a

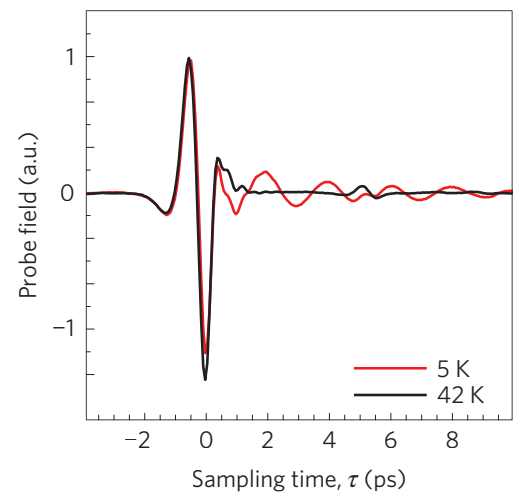

c

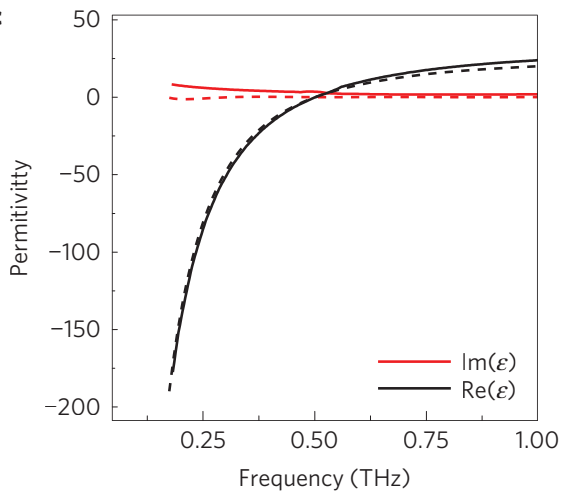

e

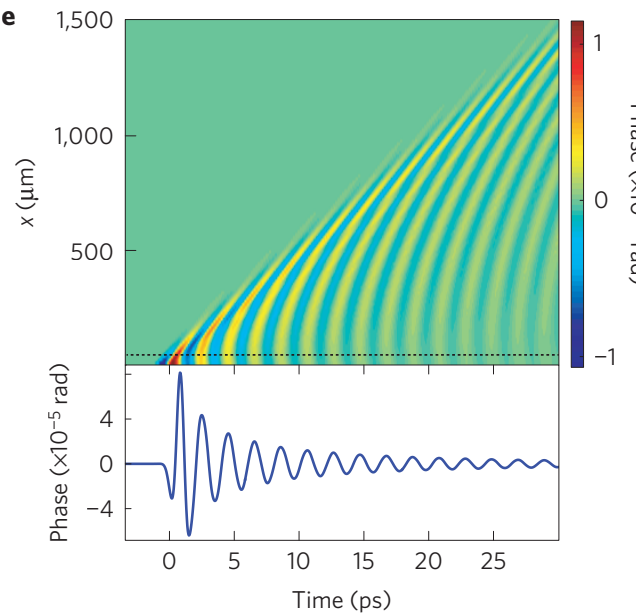

b

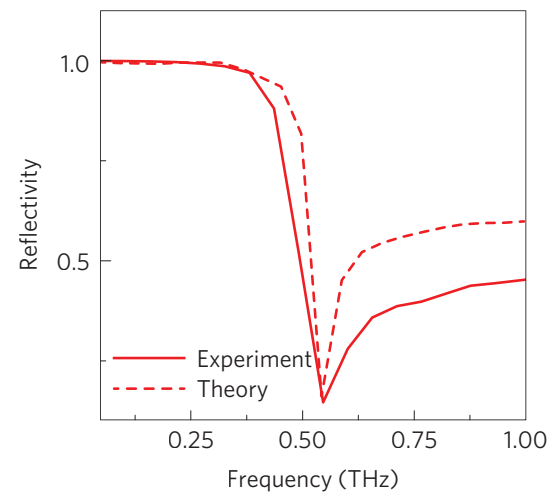

d

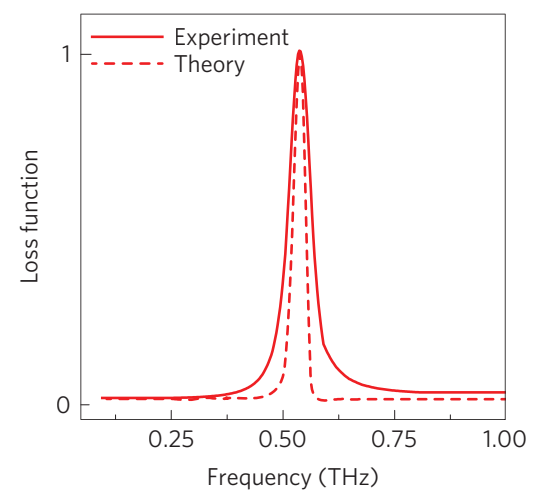

f

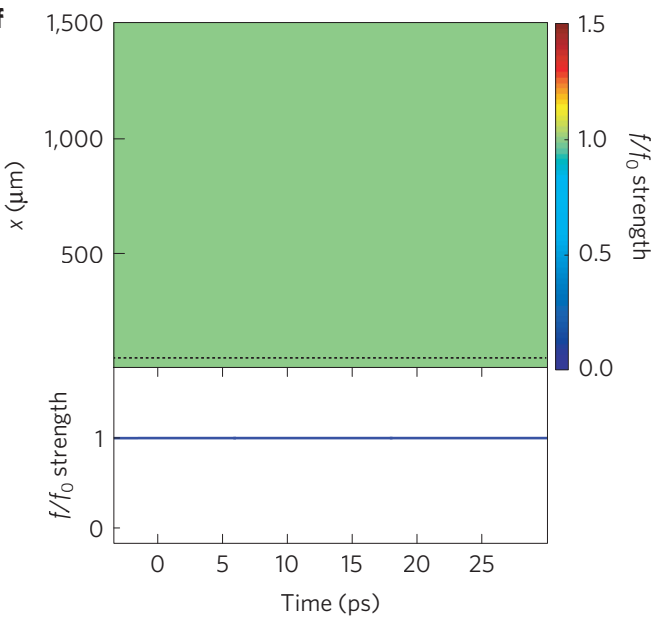

Figure 2 | Linear JPWs in $\mathrm{LBCO}_{9.5} . \mathbf{a}, E_{\text {probe }}(\tau)$ measured in the absence of a pump field both above and below $T_{\mathrm{c}}=32 \mathrm{~K}$. $\mathbf{b}$-d, Frequency-dependent, $\mathrm{c}$-axis reflectivity at $T=5 \mathrm{~K}(\mathbf{b})$ and corresponding real and imaginary part of the complex permittivity (c) and energy loss function (d). Solid lines are based on the values extracted from the $E_{\text {probe }}(\tau)$ trace of $\mathbf{a}$. Dashed lines were calculated by numerically solving the sine-Gordon equation in the linear regime. e,f, Simulated phase $\theta_{i, i+1}(x, t)$ (e) and corresponding oscillator strength $f$ (no change) (f) induced by a weak probe terahertz field. Horizontal dotted lines indicate the spatial coordinate $x$ at which the line cuts are displayed (lower panels).

implies that the oscillator strength $f(t)=f_{0} \cos \left(\theta_{0} \cos \left(\omega_{\mathrm{IP0} 0} t\right)\right) \approx f_{0}$ $\left(1-\left(\theta_{0}^{2}+\theta_{0}^{2} \cos \left(2 \omega_{\mathrm{JP} 0} t\right)\right) / 4\right)$ is modulated at a frequency $2 \omega_{\mathrm{JP} 0}$, whenever the field $E_{0}$ is large enough to make the phase excursion $\theta_{0}$ sizeable.

Figure 1 provides a pictorial representation of this physics. We plot a vector that represents both the phase difference $\theta_{i, i+1}(t)$ (vector angle) and the oscillator strength $f(t)$ (vector length).

This picture shows how, for small driving fields, only $\theta_{i, i+1}(t)$ oscillates at the driving frequency $\omega_{\mathrm{JP} 0}$, whereas for larger fields these oscillations are accompanied by a $2 \omega_{\mathrm{JP} 0}$ modulation of the oscillator strength $f(t)$.

Note also that the phenomena discussed above can be cast in terms of a Mathieu equation (see Supplementary Information 2). Thus, a $2 \omega_{\text {JP0 }}$ modulation of the oscillator strength can serve as a pump for the parametric amplification of a second, weak plasma wave at frequency $\omega_{\mathrm{JP} 0}$. In this paper we demonstrate experimentally this effect in $\mathrm{La}_{1.905} \mathrm{Ba}_{0.095} \mathrm{CuO}_{4}\left(\mathrm{LBCO}_{9.5}\right)$, a cuprate superconductor with the equilibrium JPR at $\omega_{\mathrm{JP} 0} \cong 0.5 \mathrm{THz}$.

Terahertz pulses, generated with a photoconductive antenna ${ }^{12}$, were used as a weak probe of JPWs (a schematic drawing of the measurement geometry is reported in Supplementary Information 1). A typical terahertz-field trace ${ }^{13}$ reflected by the sample is shown in Fig. 2a. Two different measurements are displayed: one taken below (red line) and the other one above (black line) the superconducting transition temperature $T_{\mathrm{c}}=32 \mathrm{~K}$. In the superconducting state, long-lived oscillations with $\sim 2$ ps period were observed on the trailing edge of the pulse, indicative of the JPR at $\omega_{\mathrm{JP} 0} \cong 0.5 \mathrm{THz}$. Figure $2 \mathrm{~b}$ (solid red line) displays the corresponding 
a

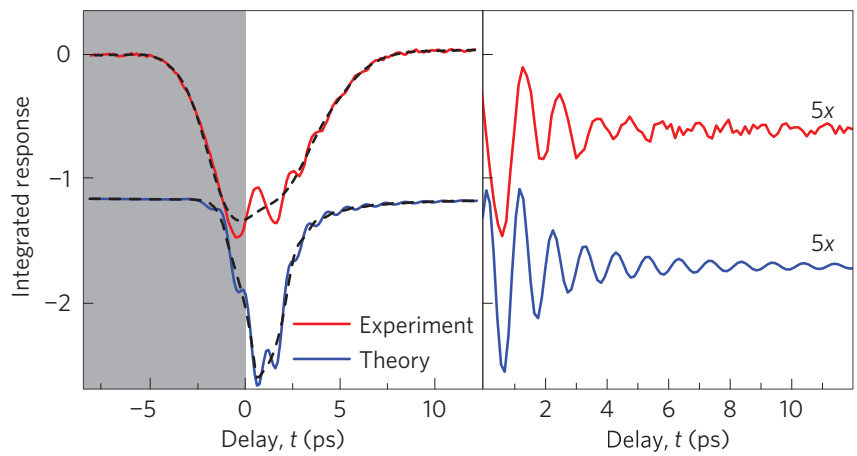

b

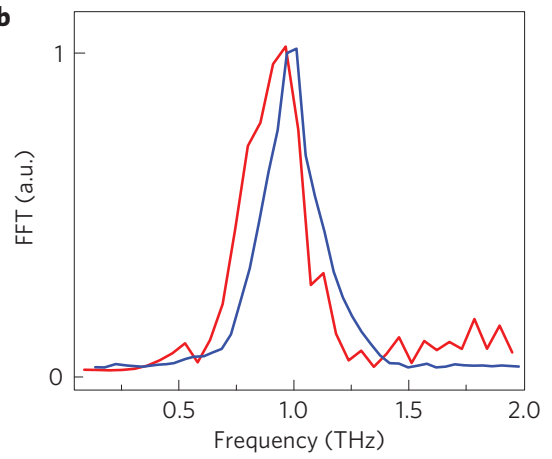

c

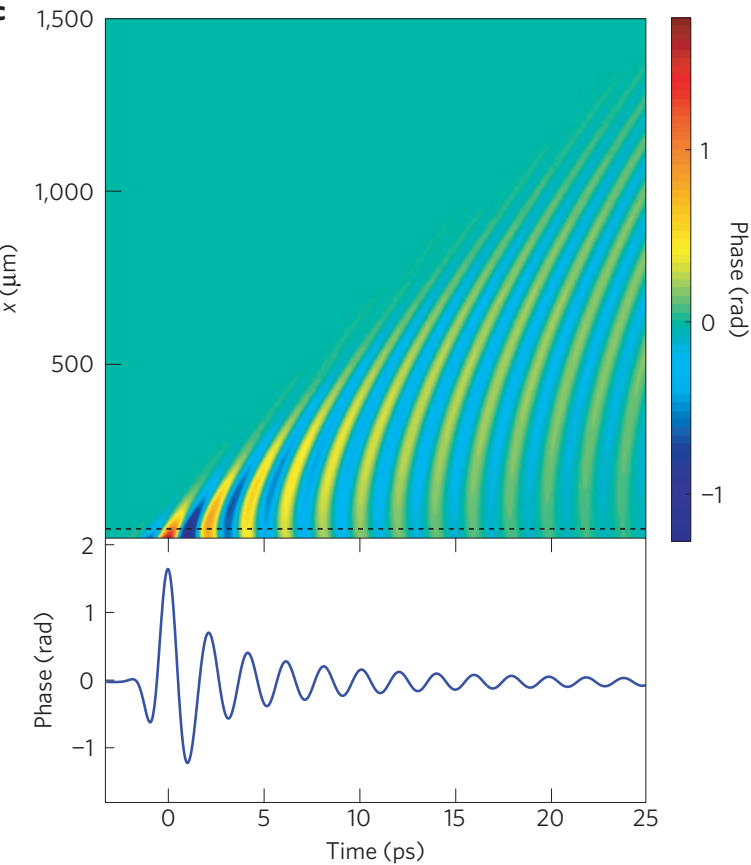

d

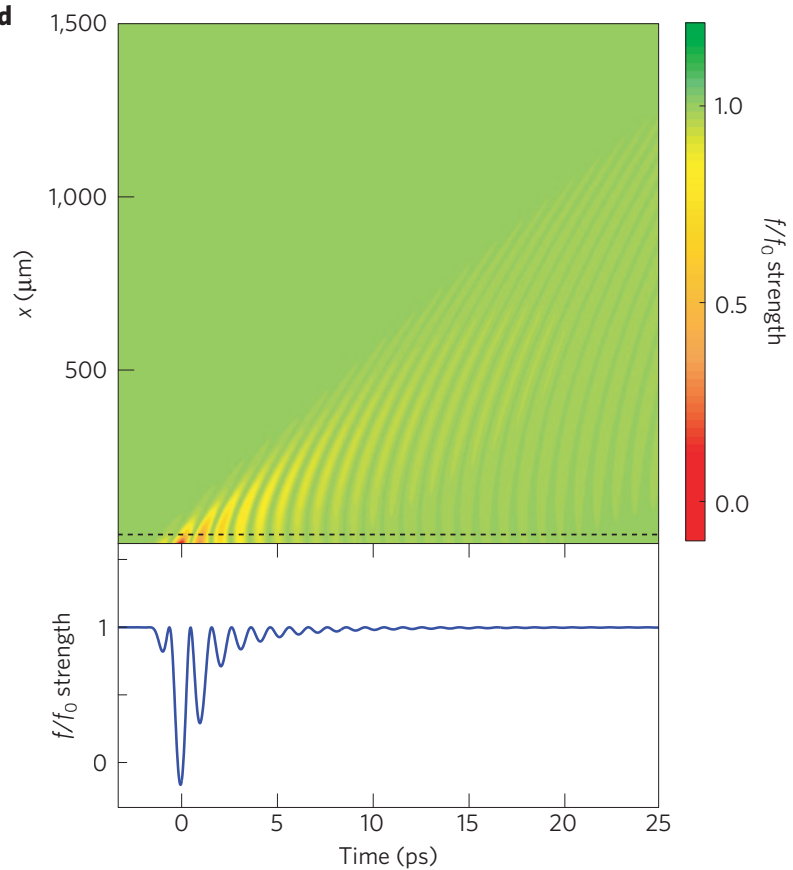

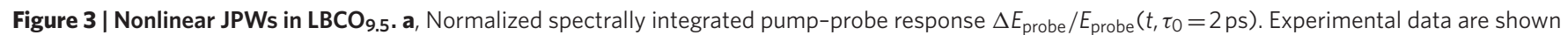
along with calculations based on the sine-Gordon equation in the nonlinear regime (displayed with a vertical offset). Dashed lines indicate an average reduction that accompanies the oscillations (see model). The reduction was subtracted through Fourier filtering ( $>0.2 \mathrm{THz}$ ) to obtain the detail of the

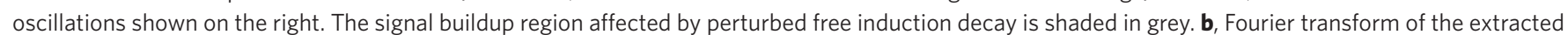
oscillations, showing a peak at $\sim 1 \mathrm{THz}$. c,d, Phase $\theta_{i, i+1}(x, t)(\mathbf{c})$ and corresponding oscillator strength $f(\mathbf{d})$ induced by a strong terahertz pump field, as determined by numerically solving the sine-Gordon equation in the nonlinear regime. Horizontal dotted lines indicate the spatial coordinate $x$ at which the line cuts are displayed (lower panels).

reflectivity edge in frequency domain. The solid lines in Fig. 2c,d are the complex dielectric permittivity $\varepsilon(\omega)$ and the loss function $L(\omega)=-\operatorname{Im}(1 / \varepsilon(\omega))=\varepsilon_{2}(\omega) /\left(\varepsilon_{1}(\omega)+\varepsilon_{2}(\omega)\right)^{2} . L(\omega)$ peaks at $\omega_{\text {JP0 }}$, where the real part of the dielectric permittivity, $\varepsilon_{1}(\omega)$, crosses zero.

These optical properties could be reproduced well by solving the wave equation in the superconductor in one dimension ${ }^{8}$ (see Supplementary Information 3 ), which yields the space- and timedependent order-parameter phase $\theta_{i, i+1}(x, t)$ (Fig. 2e) and the corresponding changes (negligible in linear response regime) of the oscillator strength $f=f_{0} \cos \left[\theta_{i, i+1}(x, t)\right]$ (Fig. 2f). The reflectivity, complex permittivity, and loss function (dashed lines in Fig. $2 \mathrm{~b}-\mathrm{d}$, respectively), calculated from these simulations by solving the electromagnetic field at the sample surface ${ }^{8}$, are in good agreement with the experimental data.

Amplification of a weak JPW like the one above (probe field) was achieved by mixing it with a second, intense pump field, which resonantly drove the Josephson phase to large amplitudes. Quasisingle cycle terahertz pulses, generated in $\mathrm{LiNbO}_{3}$ with the tilted pulse front method ${ }^{14}$ (yielding field strengths up to $\sim 100 \mathrm{kV} \mathrm{cm}^{-1}$ ), were used to excite these waves in the nonlinear regime. The spectral content of these pulses extended between 0.2 and $0.7 \mathrm{THz}$, centred at the JPR frequency (see Supplementary Information 4). Note that the pump field strength used in this experiment exceeds the expected threshold to access the nonlinear regime, defined by $\theta_{0}=\left(2 e E_{0} d\right) /\left(\hbar \omega_{\mathrm{IP0}}\right) \sim 1$ and corresponding in this material to field amplitudes $E_{0}=\left(\hbar \omega_{\mathrm{IP} 0}\right) /(2 e d) \sim 20 \mathrm{kV} \mathrm{cm}^{-1}$.

In Fig. 3, we report the time-delay-dependent, spectrally integrated pump-probe response of $\mathrm{LBCO}_{9.5}$. Changes in the reflected probe field were measured at one specific probe sampling time $\left(\tau=\tau_{0}\right)$ as a function of pump-probe time delay $(t)$. For a system in which the optical properties are dominated by a single plasma resonance, the spectrally integrated response is proportional to the plasma oscillator strength $f$.

As shown in Fig. 3a,b, this integrated response exhibits a reduction of the signal and oscillations at a frequency $\sim 2 \omega_{\mathrm{IP} 0}$. Note that the oscillation frequency did not depend on the pump electric field strength $E_{0}$, whereas the frequency reduced when the base temperature of the experiment was increased, consistent with the reduction of the equilibrium $\omega_{\mathrm{IP} 0}$ (see Supplementary Information 5 and 6). The effect completely disappeared at $T>T_{\mathrm{c}}$. 

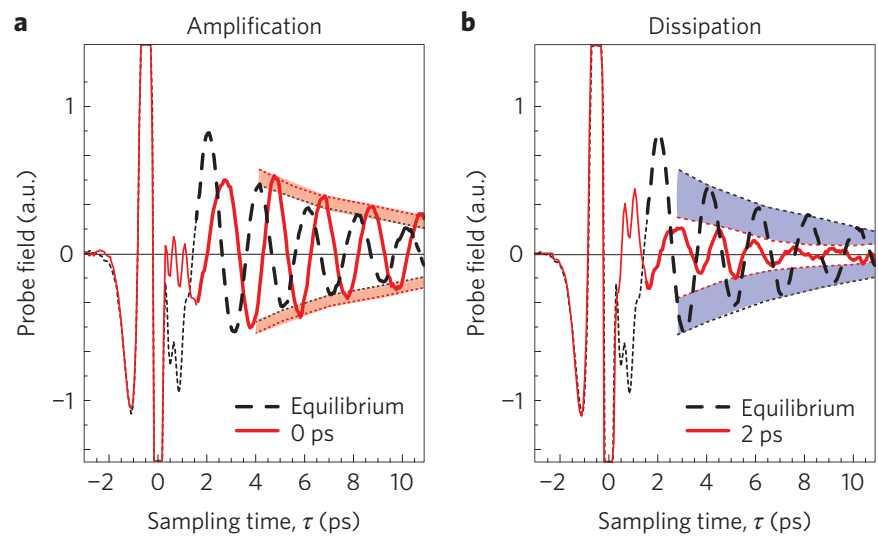

Figure 4 | Amplification and suppression of plasma oscillations. $\mathbf{a}, \mathbf{b}, E_{\text {probe }}(t, \tau)$ traces measured by scanning the electro-optic sampling time $\tau$ at selected pump-probe delays $t=0 \mathrm{ps}$ and $t=2 \mathrm{ps}$, respectively. Data are shown along with the same quantity measured at equilibrium (pump off). Plasma oscillations on the trailing edge of the pulses ( $\tau \gtrsim 2$ ps) are highlighted by thicker lines. Coloured shading indicates amplification (a) and suppression (b) of the JPW amplitude.

Hence, the theoretically predicted $2 \omega_{\mathrm{JP} 0}$ modulation of the total oscillator strength $f$ (see above) is reproduced well by the data in Fig. 3. This response could also be simulated using the spaceand time-dependent sine-Gordon equation (see Fig. 3c,d), yielding good agreement between experiment and theory (see blue lines in Fig. 3a,b).

Note that here we only analyse pump-probe delays $t \gtrsim 0 \mathrm{ps}$, because the response at the earliest times suffers from perturbed free induction decay ${ }^{15}$. This effect consists in the deformation of a coherent signal, which occurs when the pump strikes the sample during the oscillatory relaxation of the probe (for $t \lesssim 0 \mathrm{ps}$ in our case).

Selected time-domain probe traces measured before and after excitation are displayed in Fig. 4. Crucially, at specific time delays the probe field is amplified (Fig. 4a), whereas at other delays it is suppressed (Fig. 4b) with respect to that measured at equilibrium.

In Fig. 5 we report the time-delay-dependent and frequency-dependent loss function $L(t, \omega)=-\operatorname{Im}(1 / \varepsilon(t, \omega))=$ $\varepsilon_{2}(t, \omega) /\left(\varepsilon_{1}(t, \omega)+\varepsilon_{2}(t, \omega)\right)^{2}$, a quantity that peaks at the zero crossing of $\varepsilon_{1}(t, \omega)$ and is always positive for a dissipative medium (that is, a medium with $\left.\varepsilon_{2}(t, \omega)>0\right)$. The experimental data of Fig. 5a show that, after excitation, $L(\omega)$ acquires negative values around $\omega_{\mathrm{JP} 0}$ (red regions). This is indicative of a negative $\varepsilon_{2}(t, \omega)$, and hence amplification. The effect is strong near zero pump-probe time delay, then disappears after $\sim 1 \mathrm{ps}$, and is observed again periodically with a repetition frequency of $\sim 2 \omega_{\mathrm{JP} 0}$. The same effect appears also in the simulations (Fig. 5b), yielding periodic amplification at a repetition frequency of $2 \omega_{\mathrm{JP} 0}$.

In Supplementary Information 7 we report additional quantitative estimates of the degree of amplification. We include a negative absorption coefficient and a reflectivity larger than 1 at $\omega \simeq \omega_{\mathrm{JP} 0}$. The extracted values are $\alpha=(-0.090 \pm 0.003) \mu \mathrm{m}^{-1}$ and $R=(1.042 \pm 0.008)$, respectively.

The data and simulations reported here demonstrate that terahertz JPWs can be parametrically amplified, exhibiting the expected sensitivity to the relative phase of strong and weak fields mixed in this process and the oscillatory dependence at twice the frequency of the drive.

Parametric amplification of terahertz light based on nonlinear optical techniques has already been shown in the past ${ }^{16}$. However, the physics demonstrated here extend beyond potential applications in photonics, directly leading to coherent parametric control of the superfluid in layered superconductors, and providing a means to manipulate the properties of the material or to probe them in new ways ${ }^{17}$.

Moreover, the ability to amplify a plasma wave could lead to terahertz single-phonon manipulation devices that may operate above $1 \mathrm{~K}$ temperatures. These would exploit concepts that to date have been developed only at microwave frequencies and in the millikelvin regime $e^{18-21}$. Finally, the parametric phenomena discussed here can also potentially be used to squeeze $\mathrm{s}^{9,22,23}$ the superfluid phase, and may lead to control of fluctuating superconductivity ${ }^{24}$, perhaps even over a range of temperatures above $T_{c}$ (refs 25,26).
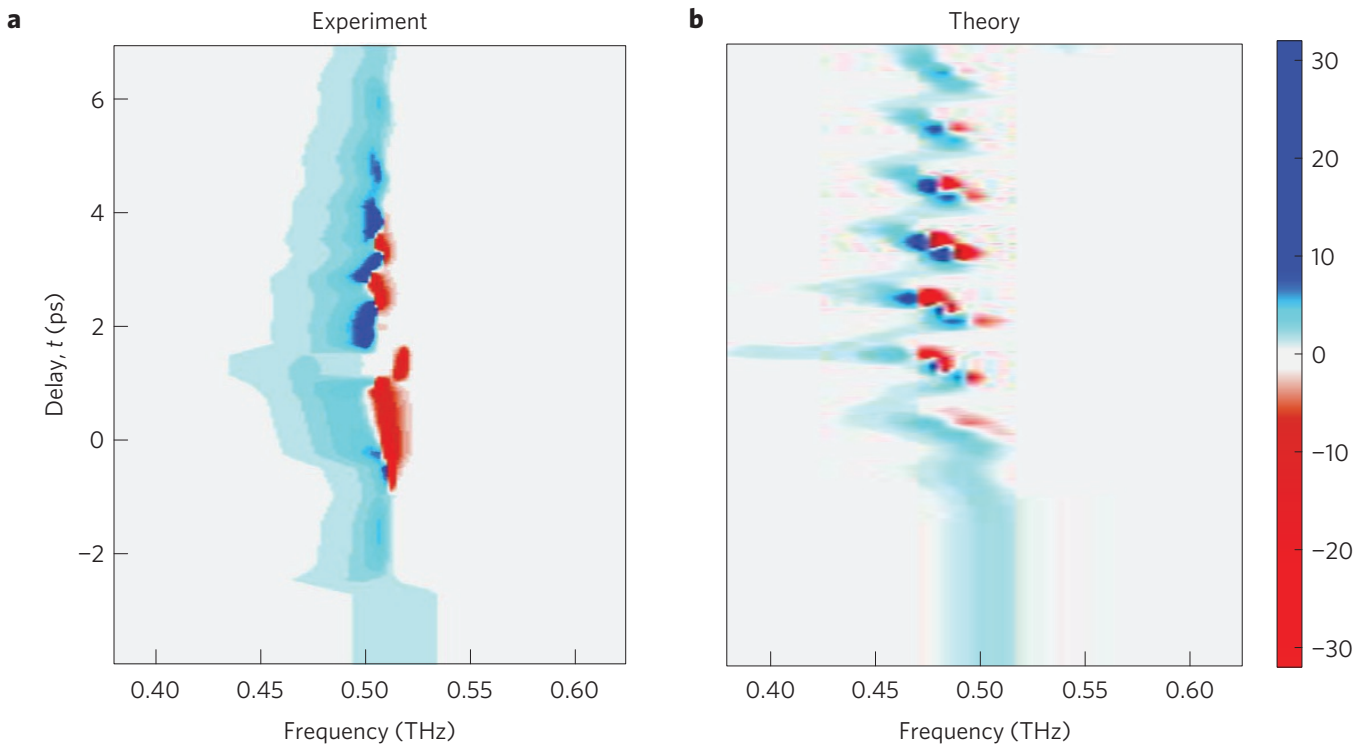

Figure 5 | Time-delay-dependent and frequency-dependent loss function. $\mathbf{a}, \mathbf{b}$, Time-delay-dependent and frequency-dependent loss function $L(t, \omega)$ determined experimentally (a) and by numerically solving the sine-Gordon equation in nonlinear regime (b). Note that experimental and simulated $L(\omega)$ in the region between $t=-4 \mathrm{ps}$ and $t=-2 \mathrm{ps}$ have been multiplied by a factor of five to be better visualized with the other data. 


\section{Methods}

Methods, including statements of data availability and any associated accession codes and references, are available in the online version of this paper.

Received 28 October 2015; accepted 13 June 2016; published online 11 July 2016

\section{References}

1. Kauranen, M. \& Zayats, A. V. Nonlinear plasmonics. Nature Photon. 6 , 737-748 (2012)

2. Basov, D. N. \& Timusk, T. Electrodynamics of high- $T_{\mathrm{c}}$ superconductors. Rev. Mod. Phys. 77, 721-779 (2005)

3. Kleiner, R., Steinmeyer, F., Kunkel, G. \& Müller, P. Intrinsic Josephson effects in $\mathrm{Bi}_{2} \mathrm{Sr}_{2} \mathrm{CaCu}_{2} \mathrm{O}_{8}$ single crystals. Phys. Rev. Lett. 68, 2394-2397 (1992).

4. Savel'ev, S., Rakhmanov, A. L., Yampol'skii, V. A. \& Nori, F. Analogues of nonlinear optics using terahertz Josephson plasma waves in layered superconductors. Nature Phys. 2, 521-525 (2006).

5. Ozyuzer, L. et al. Emission of coherent $\mathrm{THz}$ radiation from superconductors. Science 318, 1291-1293 (2007).

6. Hu, X. \& Lin, S.-Z. Phase dynamics in a stack of inductively coupled intrinsic Josephson junctions and terahertz electromagnetic radiation. Supercond. Sci. Technol. 23, 053001 (2010).

7. Dienst, A. et al. Bi-directional ultrafast electric-field gating of interlayer charge transport in a cuprate superconductor. Nature Photon. 5, 485-488 (2011).

8. Dienst, A. et al. Optical excitation of Josephson plasma solitons in a cuprate superconductor. Nature Mater. 12, 535-541 (2013).

9. Lü, X.-Y. et al. Squeezed optomechanics with phase-matched amplification and dissipation. Phys. Rev. Lett. 114, 093602 (2015).

10. Josephson, B. D. Coupled superconductors. Rev. Mod. Phys. 36, 216-220 (1964).

11. Dressel, M. \& Grüner, G. Electrodynamics of Solids: Optical Properties of Electrons in Matter (Cambridge Univ. Press, 2002).

12. Dreyhaupt, A., Winnerl, S., Dekorsy, T. \& Helm, M. High-intensity terahertz radiation from a microstructured large-area photoconductor. Appl. Phys. Lett. 86, 121114 (2005).

13. Thorsmølle, V. K. et al. C-axis Josephson plasma resonance observed in $\mathrm{Tl}_{2} \mathrm{Ba}_{2} \mathrm{CaCu}_{2} \mathrm{O}_{8}$ superconducting thin films by use of terahertz time-domain spectroscopy. Opt. Lett. 26, 1292-1294 (2001).

14. Hebling, J., Yeh, K.-L., Hoffmann, M. C., Bartal, B. \& Nelson, K. A. Generation of high-power terahertz pulses by tilted-pulse-front excitation and their application possibilities. J. Opt. Soc. Am. B 25, B6 (2008).

15. Hamm, P. Coherent effects in femtosecond infrared spectroscopy. Chem. Phys. 200, 415-429 (1995).

16. Tripathi, S. R. et al. Terahertz wave parametric amplifier. Opt. Lett. 39, 1649-1652 (2014).

17. Hohenleutner, M. et al. Real-time observation of interfering crystal electrons in high-harmonic generation. Nature 523, 572-575 (2015).
18. Vijay, R. et al. Stabilizing Rabi oscillations in a superconducting qubit using quantum feedback. Nature 490, 77-80 (2012)

19. Macklin, C. et al. A near-quantum-limited Josephson traveling-wave parametric amplifier. Science 350, 307-310 (2015).

20. Eichler, C., Salathe, Y., Mlynek, J., Schmidt, S. \& Wallraff, A. Quantum-limited amplification and entanglement in coupled nonlinear resonators. Phys. Rev. Lett. 113, 110502 (2014).

21. Castellanos-Beltran, M. A. \& Lehnert, K. W. Widely tunable parametric amplifier based on a superconducting quantum interference device array resonator. Appl. Phys. Lett. 91, 83509 (2007).

22. Almog, R., Zaitsev, S., Shtempluck, O. \& Buks, E. Noise squeezing in a nanomechanical duffing resonator. Phys. Rev. Lett. 98, 78103 (2007)

23. Zagoskin, A. M., Il'ichev, E., McCutcheon, M. W., Young, J. F. \& Nori, F. Controlled generation of squeezed states of microwave radiation in a superconducting resonant circuit. Phys. Rev. Lett. 101, 253602 (2008).

24. Denny, S. J., Clark, S. R., Laplace, Y., Cavalleri, A. \& Jaksch, D. Proposed parametric cooling of bilayer cuprate superconductors by terahertz excitation. Phys. Rev. Lett. 114, 137001 (2015).

25. Bilbro, L. S. et al. Temporal correlations of superconductivity above the transition temperature in $\mathrm{La}_{2-x} \mathrm{Sr}_{x} \mathrm{CuO}_{4}$ probed by terahertz spectroscopy. Nature Phys. 7, 298-302 (2011).

26. Corson, J., Mallozzi, R., Orenstein, J., Eckstein, J. N. \& Bozovic, I. Vanishing of phase coherence in underdoped $\mathrm{Bi}_{2} \mathrm{Sr}_{2} \mathrm{CaCu}_{2} \mathrm{O}_{8+\delta}$. Nature 398, 221-223 (1999).

\section{Acknowledgements}

The research leading to these results received funding from the European Research Council under the European Union's Seventh Framework Programme (FP7/2007-2013)/ERC Grant Agreement no. 319286 (QMAC). We acknowledge support from the Deutsche Forschungsgemeinschaft via the excellence cluster 'The Hamburg Centre for Ultrafast Imaging-Structure, Dynamics and Control of Matter at the Atomic Scale' and the Priority Program SFB925. Work performed at Brookhaven was supported by US Department of Energy, Division of Materials Science under contract no. DE-AC02-98CH10886.

\section{Author contributions}

A.C. conceived the project together with S.R. S.R. built the terahertz pump-probe experimental set-up, performed the measurement and analysed the experimental data with the support of D.N. The simulations were performed by S.R. and E.C., with input from Y.L., S.R.C. and D.J. The results were discussed and interpreted by S.R., Y.L. and A.C. The sample was grown and characterized at Brookhaven by G.D.G. The manuscript was written by A.C., S.R. and D.N., with input from all authors.

\section{Additional information}

Supplementary information is available in the online version of the paper. Reprints and permissions information is available online at www.nature.com/reprints. Correspondence and requests for materials should be addressed to A.C.

\section{Competing financial interests}

The authors declare no competing financial interests. 


\section{Methods}

Laser pulses with $100 \mathrm{fs}$ duration and $\sim 5 \mathrm{~mJ}$ energy from a commercial Ti:Sa amplifier were split into three parts $(92 \%, 7 \%, 1 \%)$. The most intense beam was used to generate strong-field terahertz pulses with energies up to $\sim 3 \mu \mathrm{J}$ via optical rectification in $\mathrm{LiNbO}_{3}$ with the tilted pulse front technique. These were collimated and then focused at normal incidence onto the sample (with polarization perpendicular to the $\mathrm{Cu}-\mathrm{O}$ planes, that is, along the $c$ axis) using a Teflon lens and a parabolic mirror, with focal lengths of $150 \mathrm{~mm}$ and $75 \mathrm{~mm}$, respectively. The pump spot diameter at the sample position was $\sim 2.5 \mathrm{~mm}$. The pump field strength was calibrated with electro-optic sampling in a $0.2-\mathrm{mm}$-thick $\mathrm{GaP}$ crystal, yielding a maximum value of $\sim 100 \mathrm{kV} \mathrm{cm}^{-1}$ (see Supplementary Information 4).

The $7 \%$ beam was used to generate the terahertz probe pulses with a photoconductive antenna. These had a dynamic bandwidth of $0.1-3 \mathrm{THz}$, corresponding to a time resolution of $\sim 250 \mathrm{fs}$. The $c$-axis optical properties of the superconductor (both at equilibrium and throughout the pump-induced dynamics) were probed in reflection geometry, with a probe incidence angle of $45^{\circ}$ and a spot diameter at the sample position of $\sim 2 \mathrm{~mm}$. The reflected probe pulses were electro-optically sampled in a 1-mm-thick ZnTe crystal, using the remaining $1 \%$ of the $800 \mathrm{~nm}$ beam. This measurement procedure returned the quantity $E_{\text {probe }}(t, \tau)$, with $t$ being the pump-probe delay and $\tau$ being the electro-optic sampling time coordinate.

The sample used in our experiment was a single crystal of $\mathrm{La}_{1.905} \mathrm{Ba}_{0.095} \mathrm{CuO}_{4}$ cut and polished along an $a c$ oriented surface $\sim 3 \times 3 \mathrm{~mm}$ in size. Its equilibrium optical response in the superconducting state was determined by measuring the complex-valued $E_{\text {probe }}(\omega)$ (pump off) both at $T<T_{\mathrm{c}}$ and $T>T_{\mathrm{c}}$, and by referencing it to the normal-state reflectivity measured in another crystal coming from the same batch of samples ${ }^{27}$.

The spectrally integrated pump-probe traces of Fig. 3 were measured by scanning the pump-probe delay $t$ at a fixed sampling time $\tau=\tau_{0}$. This was chosen to be on the trailing edge of the pulse, where the JPR oscillations are present. Note that the observed dynamics, and in particular the $2 \omega_{\text {IP0 }}$ oscillations, did not depend significantly on the specific $\tau_{0}$ value at which the scan was performed.

The frequency and time-delay-dependent loss function of Fig. 5 (as well as all complex optical properties of the perturbed material) was determined by applying Fresnel equations ${ }^{11}$ to the pump-induced changes in the reflected electric field. These were normalized by independently recording $E_{\text {probe }}(t, \tau)$ in the presence and absence of the terahertz pump field. Note that there was no need to take into account any pump-probe penetration depth mismatch in the calculation.

In the simulations, the Josephson phase evolution $\theta_{i, i+1}(x, t)$ was determined through the one-dimensional sine-Gordon equation ${ }^{6}$ :

$$
\begin{aligned}
\frac{\partial^{2} \theta_{i, i+1}(x, t)}{\partial x^{2}} & -\frac{1}{\gamma} \frac{\partial \theta_{i, i+1}(x, t)}{\partial t}-\frac{\varepsilon_{\mathrm{r}}}{c^{2}} \frac{\partial^{2} \theta_{i, i+1}(x, t)}{\partial t^{2}} \\
& =\frac{\omega_{\mathrm{JP} 0}^{2} \varepsilon_{\mathrm{r}}}{c^{2}} \sin \theta_{i, i+1}(x, t)
\end{aligned}
$$

$\gamma$ being a damping constant, $c$ the speed of light, $\varepsilon_{\mathrm{r}}$ the dielectric permittivity, and $\omega_{\mathrm{IP0}}$ the equilibrium JPR frequency. This equation was solved numerically, with the terahertz pump and probe fields overlapping at the vacuum/superconductor interface. For more details on this topic, we refer the reader to Supplementary Information 3.

Data availability. The data supporting the plots within this paper and other findings of this study are available from the corresponding author on request.

\section{References}

27. Homes, C. C. et al. Determination of the optical properties of $\mathrm{La}_{2-x} \mathrm{Ba}_{x} \mathrm{CuO}_{4}$ for several dopings, including the anomalous $x=1 / 8$ phase. Phys. Rev. $B$ 85, 134510 (2012). 